\title{
Elastic and Total Cross-Section Measurements by TOTEM
}

\section{F. Nemes* on behalf of the TOTEM collaboration \\ CERN (Also at Wigner RCP, Hungary)}

E-mail: fnemes@cern.ch

The TOTEM experiment at the LHC has measured proton-proton (pp) elastic scattering in dedicated runs at $\sqrt{s}=2.76,7,8$ and $13 \mathrm{TeV}$ centre-of-mass energies. The total, elastic and inelastic pp cross-sections have been derived for each energy using the optical theorem and the luminosity independent method. At $13 \mathrm{TeV}$, the total pp cross-section has also been derived for the first time at LHC using the Coulomb amplitude for the normalization of the elastic $\mathrm{d} \sigma / \mathrm{d} t$. TOTEM has excluded using $\sqrt{s}=8 \mathrm{TeV}$ data a purely exponential nuclear differential cross-section $\mathrm{d} \sigma / \mathrm{d} t$ at low $|t|$ for elastic pp scattering. The deviation has been confirmed at $\sqrt{s}=13 \mathrm{TeV}$. The $\rho$ parameter, the real to imaginary ratio of the nuclear elastic scattering amplitude at $t=0$, has been measured precisely at $\sqrt{s}=13 \mathrm{TeV}$ using the Coulomb-nuclear interference region. To properly describe the measured $\rho$ value and all the TOTEM $\sigma_{\text {tot }}$ measurements, in addition to the exchange of photons and colourless two-gluon compound states, the exchange of a colourless C-odd three-gluon compound state in the $t$-channel should be added for elastic pp scattering. At all LHC energies, TOTEM has observed a diffractive minimum (“dip") followed by a secondary maximum ("bump") in the elastic pp $\mathrm{d} \sigma / \mathrm{d} t$. In the measurement of the D0 experiment at $\sqrt{s}=1.96 \mathrm{TeV}$, no dip or bump can be observed in the elastic proton-antiproton $\mathrm{d} \sigma / \mathrm{d} t$. Under the assumption that possible effects due to the energy difference between the $2.76 \mathrm{TeV}$ TOTEM and the $1.96 \mathrm{TeV}$ D0 measurements can be neglected, the results provide evidence for the exchange of a colourless C-odd 3-gluon compound state in the $t$-channel of proton-(anti)proton elastic scattering.

XXV International Workshop on Deep-Inelastic Scattering and Related Subjects 8-12 April, 2019

Torino, Italy

\footnotetext{
* Speaker.
} 


\section{INTRODUCTION}

The TOTEM (TOTal cross section, Elastic scattering and diffraction dissociation Measurement at the LHC) experiment has been designed to measure the total proton-proton (pp) cross-section, elastic scattering and diffractive processes at the LHC [1].

The experimental apparatus of TOTEM is composed of three subdetectors: the Roman Pots (RP), the T1 and the T2 inelastic forward telescopes. The detectors are placed symmetrically on both sides of the Interaction Point 5 (IP5), which is shared with the CMS experiment.

The RPs are moveable beam-pipe insertions, hosting edgeless silicon detectors to detect leading protons scattered at very small angles. In order to maximize the acceptance of the experiment for elastically scattered protons, the RPs are able to approach the beam center to a transverse distance as small as $1 \mathrm{~mm}$. The alignment of the RPs is optimized by reconstructing common tracks going through the overlap between the vertical and horizontal RPs [1,2].

The T1 telescope is based on cathode strip chambers placed at $\pm 9 \mathrm{~m}$ and covers the pseudorapidity range $3.1 \leq|\eta| \leq 4.7$; the $\mathrm{T} 2$ telescope is based on gas electron multiplier chambers placed at $\pm 13.5 \mathrm{~m}$ and covers the pseudorapidity range $5.3 \leq|\eta| \leq 6.5$. The pseudorapidity coverage of the two telescopes at $\sqrt{s}=2.76,7,8$ and $13 \mathrm{TeV}$ allows the detection of about $96 \%, 95 \%, 94 \%$ and $92 \%$, respectively, of the inelastic pp collisions, including collisions producing diffractive mass above about $2.1 \mathrm{GeV}, 3.4 \mathrm{GeV}, 3.6 \mathrm{GeV}$ and $4.6 \mathrm{GeV}$, respectively $[11,13,16]$.

Before the LHC long shutdown one (LS1) the RPs, used for the measurements, were located at distances of 215-220 m from IP5 [1]. The actual layout, i.e., after the LHC LS1, is different in RP location and quantity. The RP stations previously installed at $\pm 147 \mathrm{~m}$, from IP5, have been relocated to $\pm 210 \mathrm{~m}$. Moreover, newly designed horizontal RPs have been installed between the two units of the $\pm 220 \mathrm{~m}$ station $[3,4]$.

\section{ELASTIC SCATTERING AND TOTAL CROSS-SECTION MEASUREMENTS}

For each tagged elastic event the four-momentum transfer squared $t$ is reconstructed using the LHC optical functions, characterized with the so-called betatron amplitude at IP5 $\beta^{*}$ [1]. The TOTEM experiment developed a novel experimental method to estimate the optical functions at the RP locations, using the measured elastically scattered protons $[5,6]$.

The total inelastic rate $N_{\text {inel }}$, measured by the T1 and T2 telescopes, and the total nuclear elastic rate $N_{\mathrm{el}}$ with its extrapolation to zero four-momentum transfer squared $t=0$ are combined with the optical theorem to obtain the total cross-section in a luminosity, $\mathscr{L}$, independent way

$$
\sigma_{\mathrm{tot}}=\left.\frac{16 \pi}{1+\rho^{2}} \cdot \frac{\mathrm{d} N_{\mathrm{el}}}{\mathrm{d} t}\right|_{t=0} \cdot\left(N_{\mathrm{el}}+N_{\mathrm{inel}}\right)^{-1}
$$

The measured elastic $N_{\mathrm{el}}$ and inelastic rates $N_{\text {inel }}$ allow for the determination of the elastic and inelastic cross-sections as well.

The TOTEM experiment determined the total pp cross-section at $\sqrt{s}=7 \mathrm{TeV}$ using the luminosity independent method [12], which was shown to be consistent with the total cross-sections 


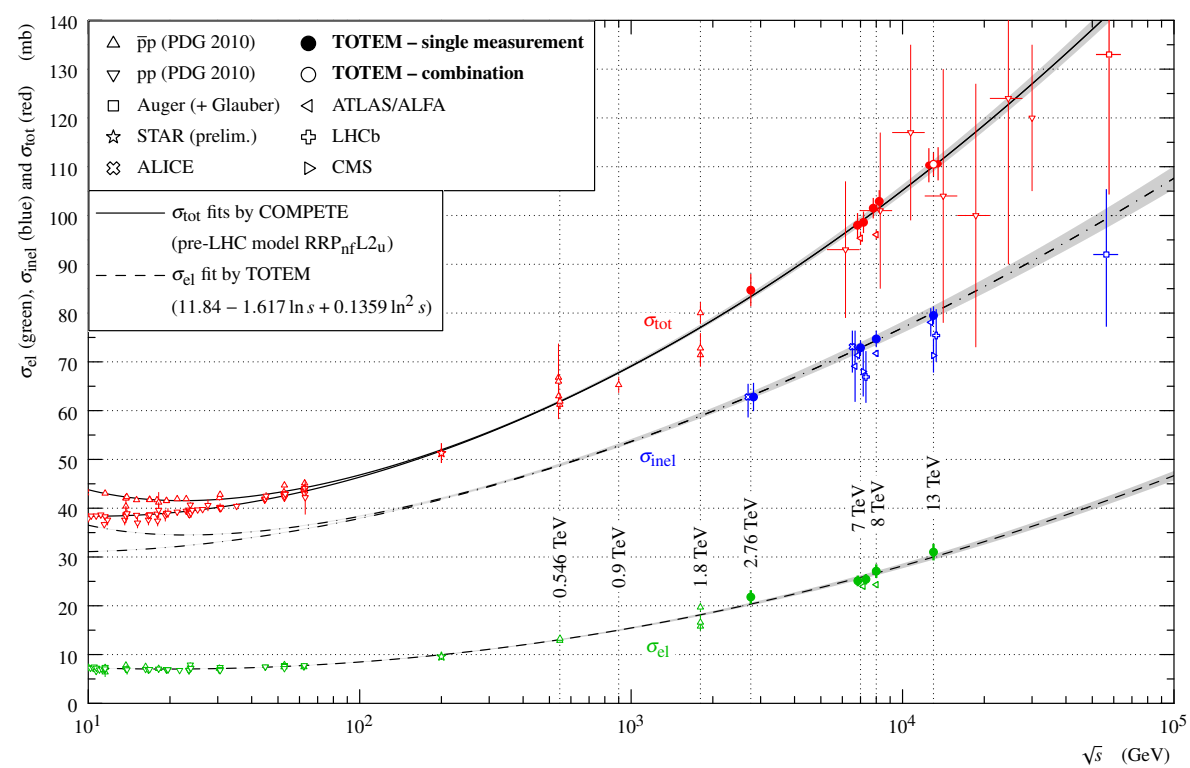

Figure 1: A compilation of total, inelastic and elastic pp cross-section measurements, see Ref. [7, 20] and references therein. The red points indicate the TOTEM total cross-section measurements at $\sqrt{s}=2.76,7,8$ and $13 \mathrm{TeV}$. These are compared to pre-LHC models using low energy data for the extrapolation to LHC [8].

measured in independent ways, see Table 1 . The elastic and inelastic cross-sections were found to be $\sigma_{\mathrm{el}}=(25.1 \pm 1.1) \mathrm{mb}$ and $\sigma_{\text {inel }}=(72.9 \pm 1.5) \mathrm{mb}$, respectively.

\begin{tabular}{|c|c|c|c|c|}
\hline Method & $\mathscr{L}$ independent [12] & Elastic only [9] & Elastic only [10] & $\rho$ independent [12] \\
\hline$\sigma_{\text {tot }}[\mathrm{mb}]$ & $98.0 \pm 2.5$ & $98.3 \pm 2.8$ & $98.6 \pm 2.2$ & $99.1 \pm 4.3$ \\
\hline
\end{tabular}

Table 1: The total cross-section $\sigma_{\text {tot }}$ results measured by the TOTEM experiment at $\sqrt{s}=7 \mathrm{TeV}$ with three different methods and two different data sets.

The luminosity-independent measurements were repeated at $\sqrt{s}=2.76,8$ and $13 \mathrm{TeV}$. At $\sqrt{s}=2.76 \mathrm{TeV}$, the total cross-section was found to be $\sigma_{\mathrm{tot}}=(84.7 \pm 3.3) \mathrm{mb}$, while the elastic and inelastic cross-section were $\sigma_{\mathrm{el}}=(21.8 \pm 1.4) \mathrm{mb}$ and $\sigma_{\text {inel }}=(62.8 \pm 2.9) \mathrm{mb}$, respectively [16]. At $\sqrt{s}=8 \mathrm{TeV}$, the total, elastic and inelastic cross-sections of $\sigma_{\mathrm{tot}}=(101.7 \pm 2.9) \mathrm{mb}, \sigma_{\mathrm{el}}=$ $(27.1 \pm 1.4) \mathrm{mb}$ and $\sigma_{\text {inel }}=(74.7 \pm 1.7) \mathrm{mb}$, respectively, were obtained [13]. Finally at $\sqrt{s}=$ $13 \mathrm{TeV}$, the total, elastic and inelastic cross-sections were found to be $\sigma_{\mathrm{tot}}=(110.6 \pm 3.4) \mathrm{mb}$, $\sigma_{\mathrm{el}}=(31.0 \pm 1.7) \mathrm{mb}$ and $\sigma_{\text {inel }}=(79.5 \pm 1.8) \mathrm{mb}$, respectively [16].

In 2016, TOTEM took data during a special run with $\beta^{*}=2500 \mathrm{~m}$ optics at $13 \mathrm{TeV}$ collision energy, which allowed to probe sufficiently low $|t|$-values to be sensitive to the Coulomb amplitude allowing a first total pp cross section measurement at LHC with Columb normalization $\sigma_{\mathrm{tot}}=(110.3 \pm 3.5) \mathrm{mb}[20]$. Combining the two uncorrelated TOTEM measurement at $13 \mathrm{TeV}$, luminosity independent and Coulomb normalization, yields $\sigma_{\mathrm{tot}}=(110.5 \pm 2.4) \mathrm{mb}$. Fig. 1 shows a compilation of all the results together with other LHC measurements. The observed cross-sections are in agreement with the extrapolation of low-energy data to LHC [8] and cosmic ray results.

Thanks to a high statistics $\beta^{*}=90 \mathrm{~m}$ data set at $\sqrt{s}=8 \mathrm{TeV}$ energy, the TOTEM experiment excluded a purely exponential elastic pp differential cross-section [14]. The significance of the 
exclusion is greater than $7 \sigma$ in the $|t|$ range from 0.027 to $0.2 \mathrm{GeV}^{2}$. Using refined parametrizations for the extrapolation to the optical point, $t=0$, yielded total cross-section values $\sigma_{\text {tot }}=(101.5 \pm$ $2.1) \mathrm{mb}$ and $\sigma_{\mathrm{tot}}=(101.9 \pm 2.1) \mathrm{mb}$, compatible with the previous measurements. The deviation from the purely exponential elastic pp differential cross-section has been confirmed at $13 \mathrm{TeV}$ [19].

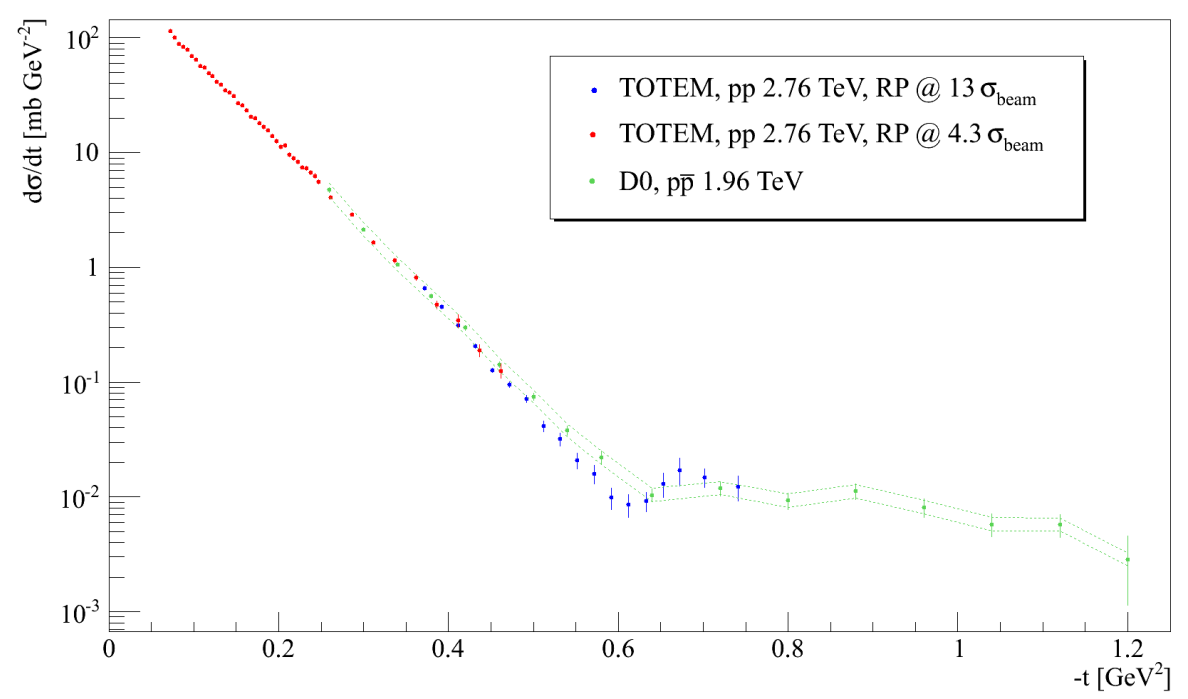

Figure 2: The differential elastic pp crosssection $\mathrm{d} \sigma / \mathrm{d} t$ at $\sqrt{s}=2.76 \mathrm{TeV}$ measured by the TOTEM experiment and the elastic p $\bar{p}$ cross-section measurement of the D0 experiment at $1.96 \mathrm{TeV}$ [18]. The green dashed line indicates the normalization uncertainty of the D0 measurement.

The TOTEM experiment performed its first measurement of elastic scattering in the Coulombnuclear interference region using data collected at $\sqrt{s}=8 \mathrm{TeV}$ with a special beam optics of $\beta^{*}=1000 \mathrm{~m}$ in 2012 [15]. The $\rho$ parameter was for the first time at LHC extracted via the Coulomb-nuclear interference, and was found to be $\rho=0.12 \pm 0.03$. Removing the Coulombnuclear interference contribution when extrapolating to the optical point, $t=0$, yielded total crosssection values of $\sigma_{\mathrm{tot}}=(102.9 \pm 2.3) \mathrm{mb}$ and $\sigma_{\mathrm{tot}}=(103.0 \pm 2.3) \mathrm{mb}$ for central and peripheral phase descriptions, respectively, compatible with the previous total cross-section measurements.

The $\beta^{*}=2500 \mathrm{~m}$ optics run at $\sqrt{s}=13 \mathrm{TeV}$ with high statistics allowed also for a precise measurement of $\rho$, giving $\rho=0.09 \pm 0.01$ and $\rho=0.10 \pm 0.01$, depending on the different physics assumptions and mathematical modelling. These $\rho$ results combined with all the TOTEM $\sigma_{\text {tot }}$ measurements indicate that it is not sufficient to include only photon and colourless 2-gluon compound state exchange, the so-called Pomeron, in the $t$-channel to properly describe elastic pp scattering. A significantly better description is obtained both in Regge-like frameworks and QCD by adding also the exchange of a colourless C-odd 3-gluon compound state [20], the so-called Odderon. On the contrary, if shown that the exchange of a C-odd 3-gluon compound state in the $t$-channel is not of importance for the description of elastic pp scattering at low $|t|$, the $\rho$ value determined by TOTEM would represent a first evidence of a slowing down of the total cross-section growth at higher energies.

At $13 \mathrm{TeV}$, the differential cross-section has been measured in the $[0.04 ; 4] \mathrm{GeV}^{2}$ range of $t$ using a very-high statistics sample (more than $10^{9}$ ) of elastic events taken in 2015 using a dedicated data acquisition system allowing an increased data taking rate by an order of magnitude. This sam- 
ple allowed for a precise measurement of the non-exponential part that contains a diffractive minimum ("dip"), a secondary maximum ("bump") and a non-exponential tail without further structure, see Fig. 3 [19]. The dip position at $13 \mathrm{TeV}$ was found to be $\left|t_{\text {dip }}\right|=\left(0.47 \pm 0.004^{\text {stat }} \pm 0.01^{\text {syst }}\right) \mathrm{GeV}^{2}$ and the ratio of the $\mathrm{d} \sigma / \mathrm{d} t$ at the bump and at the $\operatorname{dip} R=1.77 \pm 0.01$. Using $\beta^{*}=11 \mathrm{~m}$ optics data taken in 2013, also the dip at $\sqrt{s}=2.76 \mathrm{TeV}$ could be observed; the dip position was $\left|t_{\mathrm{dip}}\right|=(0.61 \pm 0.03) \mathrm{GeV}^{2}$ and the bump-dip cross-section ratio $R=1.7 \pm 0.2$, as shown in Fig. 2 [17]. These new results confirm the $\mathrm{d} \sigma / \mathrm{d} t$ feature of a dip and a bump at TeV scale already observed at $7 \mathrm{TeV}$ with a dip position $\left|t_{\text {dip }}\right|=\left(0.53 \pm 0.01^{\text {stat }} \pm 0.01^{\text {syst }}\right) \mathrm{GeV}^{2}$ and a bumpdip cross-section ratio $R=1.7 \pm 0.1[2,20]$. The series of TOTEM elastic pp measurements show that the dip is a permanent feature of the pp differential cross-section at TeV scale.

When the $2.76 \mathrm{TeV} \mathrm{d} \sigma / \mathrm{d} t$ measurement of TOTEM is compared to the proton-antiproton $(\mathrm{p} \overline{\mathrm{p}})$ measurement of the D0 experiment at $\sqrt{s}=1.96 \mathrm{TeV}$, a significant difference can be observed, see Fig. 2. Under the assumption that possible effects due to the energy difference between TOTEM and D0 can be neglected, the result provides evidence for the exchange of a colourless C-odd 3 -gluon compound state in the $t$-channel of pp and $\mathrm{p} \overline{\mathrm{p}}$ elastic scattering.

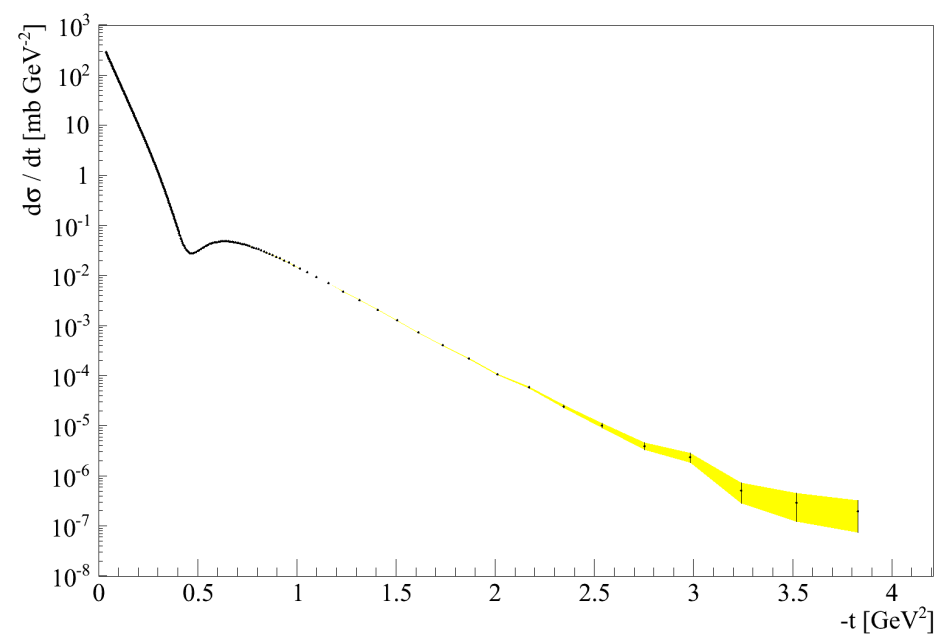

Figure 3: Differential elastic pp cross-section $\mathrm{d} \sigma / \mathrm{d} t$ at $\sqrt{s}=13 \mathrm{TeV}$. The statistical and $|t|$-dependent correlated systematic uncertainty envelope is shown as a yellow band.

\section{CONCLUSIONS}

The TOTEM experiment has measured elastic pp scattering at $\sqrt{s}=2.76,7,8$ and $13 \mathrm{TeV}$. The total, elastic and inelastic pp cross-sections have been derived for all energies using the optical theorem and the luminosity independent method. At $\sqrt{s}=8 \mathrm{TeV}$, TOTEM has also excluded a purely exponential nuclear pp differential cross-section at low $|t|$. This deviation has been confirmed at $13 \mathrm{TeV}$. At $13 \mathrm{TeV}$, the $\rho$ parameter has been precisely measured and the total pp cross-section using the Coulomb amplitude has been derived for the first time at the LHC. The $\rho$ measurement combined with all the TOTEM $\sigma_{\text {tot }}$ measurements indicate the necessity to add the exchange of a colourless C-odd 3-gluon compound state in the $t$-channel of elastic pp scattering. 
At $\sqrt{s}=2.76 \mathrm{TeV}$, a diffractive minimum "dip" and a secondary maximum "bump" has been observed; when compared to the p $\bar{p}$ measurement of the D0 experiment at $\sqrt{s}=1.96 \mathrm{TeV}$, a significant difference can be observed. Under the assumption that possible effects due to the energy difference between TOTEM and D0 can be neglected, the result provides evidence for the exchange

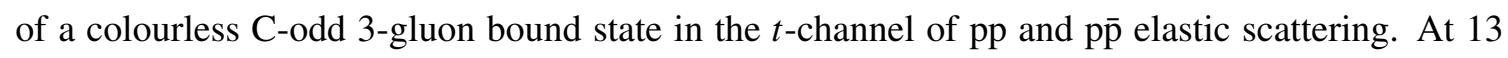
$\mathrm{TeV}$, the differential cross-section has been measured in the $\left[0.04 \mathrm{GeV}^{2} ; 4 \mathrm{GeV}^{2}\right]|t|$-range allowing for the precise measurement of the dip. The series of TOTEM elastic pp measurements show that the dip is a permanent feature of the pp differential cross-section at the $\mathrm{TeV}$ scale.

\section{References}

[1] G. Anelli et al. [TOTEM Coll.], JINST 3 (2008) S08007.

[2] G. Antchev et al. [TOTEM Coll.], Europhys. Lett. 95 (2011) 41001.

[3] TOTEM Coll. [TOTEM Coll.], CERN-LHCC-2013-009, LHCC-P-007.

[4] M. Albrow et al. [CMS and TOTEM Coll.], CERN-LHCC-2014-021, TOTEM-TDR-003, CMS-TDR-13.

[5] G. Antchev et al. [TOTEM Coll.], New J. Phys. 16 (2014) 103041.

[6] F. Nemes, H. Niewiadomski and H. Burkhardt, Conf. Proc. C 1205201 (2012) 136.

[7] M. Tanabashi et al. [PDG Coll.], Phys. Rev. D 98 (2018) 030001.

[8] J. R. Cudell et al. [COMPETE Coll.], Phys. Rev. Lett 89 (2002) 201801.

[9] G. Antchev et al. [TOTEM Coll.], Europhys. Lett. 96 (2011) 21002.

[10] G. Antchev et al. [TOTEM Coll.], Europhys. Lett. 101 (2013) 21002.

[11] G. Antchev et al. [TOTEM Coll.], Europhys. Lett. 101 (2013) 21003.

[12] G. Antchev et al. [TOTEM Coll.], Europhys. Lett. 101 (2013) 21004.

[13] G. Antchev et al. [TOTEM Coll.], Phys. Rev. Lett. 111 (2013) 012001.

[14] G. Antchev et al. [TOTEM Coll.], Nucl. Phys. B 899 (2015) 527.

[15] G. Antchev et al. [TOTEM Coll.], Eur. Phys. J. C 76 (2016) 661.

[16] G. Antchev et al. [TOTEM Coll.], Eur. Phys. J. C 79 (2019) 103.

[17] G. Antchev et al. [TOTEM Coll.], arXiv:1812.08610 [hep-ex].

[18] V. M. Abazov et al. [D0 Coll.], Phys. Rev. D 86 (2012) 012009.

[19] G. Antchev et al. [TOTEM Coll.], arXiv:1812.08283 [hep-ex].

[20] G. Antchev et al. [TOTEM Coll.], arXiv:1812.04732 [hep-ex]. 